\title{
In Vitro Activity of Carbapenems Alone and in Combination With Amikacin Against KPC-Producing Klebsiella Pneumoniae
}

\author{
Jennifer Le ${ }^{\mathrm{a}, \mathrm{b}, \mathrm{e}}$, Barbara McKee ${ }^{\mathrm{b}}$, Warunee Srisupha-Olarn ${ }^{\mathrm{c}, \mathrm{d}}$, David S. Burgess ${ }^{\mathrm{c}, \mathrm{d}}$
}

\begin{abstract}
Background: Although carbapenems are the primary treatment strategy for invasive infections caused by ESBL bacteria, case reports of these pathogens with reduced carbapenem susceptibility have emerged. One potential treatment modality is to optimize the use of anti-infectives with combination therapy. We evaluated the activity of carbapenems alone and in combination with amikacin against these clinical isolates.
\end{abstract}

Methods: Time-kill studies evaluated ertapenem (ETP), imipenem (IPM), meropenem (MEM), and amikacin (AMK) against 4 nonduplicate clinical isolates of Klebsiella pneumoniae that were resistant to these antibiotics. Synergy was defined as $\geq 2 \log _{10}$ decrease $\mathrm{CFU} / \mathrm{mL}$ at $24 \mathrm{~h}$ for the combination when compared with the most active single agent of the combination, plus the number of surviving organisms for the antimicrobial combination was $\geq 2 \log _{10}$ less than the initial inoculum.

Results: All isolates carried bla $_{K P C-3}$ and genes encoding TEM-1 and SHV-11/-36; and were resistant to carbapenems (MIC at $\geq 8$

Manuscript accepted for publication April 28, 2011

${ }^{a}$ University of California, San Diego, Skaggs School of Pharmacy and Pharmaceutical Sciences, 9500 Gilman Drive, MC 0714, La Jolla, CA 92093-0714, USA

${ }^{\mathrm{b}}$ Long Beach Memorial Medical Center, 2801 Atlantic Ave, PO Box 1428, Long Beach, CA 90801-1428, USA

${ }^{c}$ Center for Advancement of Research and Education in Infectious Diseases, University of Texas, College of Pharmacy, Austin, TX, USA

${ }^{\mathrm{d}}$ Pharmacotherapy Education and Research Center, School of Medicine, The University of Texas Health Science Center San Antonio, 7703 Floyd Curl Drive- MSC 6220, San Antonio, Texas 78229-3900, USA

eCorresponding author: Jennifer Le, Email: jenle@ucsd.edu

doi:10.4021/jocmr551w $\mu \mathrm{g} / \mathrm{mL}$ for ETP, MEM and IPM) and AMK (MIC $32 \mu \mathrm{g} / \mathrm{mL}$ ) using broth microdilution. As monotherapy, none of the carbapenems nor AMK achieved and maintained bactericidal activity defined as $\geq 99.9 \%$ or $>3 \log _{10}$ killing. From time-kill studies, synergy was demonstrated for MEM and IPM in combination with AMK over the entire $24 \mathrm{~h}$ against all isolates. In addition, MEM and IPM with AMK achieved and maintained bactericidal activity ( $\geq 99.9 \%$ killing) at $24 \mathrm{~h}$ against 2 and 1 isolate(s), respectively. Bactericidal activity and synergy were not observed for ETP combinations.

Conclusions: The combination of MEM or IPM with AMK displayed synergistic activity against KPC-3-producing K. pneumoniae isolates.

Keywords: ESBL; Klebsiella pneumoniae; KPC; Carbapenemase; Time-kill; Meropenem; Amikacin; Imipenem; Ertapenem; Carbapenem; Synergy

\section{Introduction}

Carbapenems are the primary treatment strategy for serious infections caused by extended-spectrum beta-lactamase (ESBL)-producing Klebsiella pneumoniae. However, production of KPC $\beta$-lactamase, a serine carbapenemase primarily detected in K. pneumoniae, has emerged in the USA and is probably contributing to carbapenem resistance rates among Enterobacteriaceae $[1,2]$.

In light of this carbapenem resistance and with the dearth of new antimicrobial agents with novel mechanisms of action, combination therapy using existing antibiotics becomes an attractive option. The combination of two antibiotics from different classes will increase the probability that at least one antibiotic will provide adequate activity against the multi-drug resistance isolate and enhance their activity when synergy is present. This is crucial since inappropriate initial antimicrobial therapy has been associated with increased mortality [3-5].

Based on limited clinical experience, susceptibility data and single antibiotic pharmacokinetic-pharmacodynamic studies, polymixin B [6, 7], tigecycline [6-9], AMK [6], carbapenems [10-12], and clavulanic acid like ticarcillin/ 
clavulanate [13] may play a role in combination therapy for infections caused by KPC-producing pathogens. We performed time-kill studies to evaluate the in vitro activity of carbapenems [including ertapenem (ETP), imipenem (IPM) and meropenem (MEM)] alone or in combination with amikacin (AMK) against KPC-producing K. pneumoniae. These antibiotics were selected due to their safety profile and feasibility to be used empirically since detection of KPC production in clinical laboratory can be challenging. In addition, amikacin has a fast rate of bactericidal activity [14] and postantibiotic effect [15].

\section{Materials and Methods}

Drug stock solutions were prepared according to the manufacturer's recommendations from powders of ETP (AstraZeneca, Wilmington, DE), IPM (Merck, Whitehouse Station, NJ), MEM (AstraZeneca, Wilmington, DE), and AMK (Bristol-Myers Squibb, New York, NY). Drug concentrations were based on the mean steady-state serum concentration obtained using normal doses of each antibiotic in normal healthy volunteers. The following antibiotics at the specified concentrations $(\mu \mathrm{g} / \mathrm{mL})$ were used: ETP 2, IPM 4, MEM 4, and AMK 16.

Four non-duplicate isolates of $K$. pneumoniae obtained from an observational clinical outcomes study carried $b l a_{K-}$ ${ }_{P C-3}$ and genes encoding TEM-1 and SHV-11/-36 [16]. Isolate number $16,17,38$ and 50 corresponded to the following patients 1, 2, 4 and 3, respectively. Isolates were negative for all other beta-lactamase encoding genes, including plasmidic AmpC, serine- and metallo- $\beta$-lactamases, tested. Molecular typing demonstrated that all 4 KPC-3-producing strains were clonally related.

Using broth microdilution as described by the Clinical and Laboratory Standards Institute (CLSI), all strains were resistant to carbapenems (MIC at $\geq 8 \mu \mathrm{g} / \mathrm{mL}$ for ETP, MEM and IPM) and AMK (MIC $32 \mu \mathrm{g} / \mathrm{mL}$ ). Likewise using Etests, MIC values of all carbapenems were $\geq 32 \mu \mathrm{g} / \mathrm{mL}$ for all isolates and AMK were 128, 32, 48 and $48 \mu \mathrm{g} / \mathrm{mL}$ for $\mathrm{C} 16$, C17, C38 and C50, respectively.

Time-kill studies were performed using a final volume of $25 \mathrm{~mL}$ of cation-adjusted Mueller-Hinton broth and an initial inoculum of $\sim 1 \times 10^{6} \mathrm{CFU} / \mathrm{mL}$. Samples were taken at 0 , 4, 8, 12 and $24 \mathrm{~h}$. Fifty $\mu \mathrm{L}$ was plated on trypticase soy agar plates using a spiral plater (Spiral Biotech, Bethesda, MD) and then incubated at $35^{\circ} \mathrm{C}$ for $24 \mathrm{~h}$ before colony counts were determined using a laser colony counter (CASBA 4, Spiral Biotech, Bethesda, MD). The spiral plater controlled antibiotic carry-over [17]. Time-kill profiles were constructed ( $\log \mathrm{CFU} / \mathrm{mL}$ versus time). The limit of quantification was $10^{2} \mathrm{CFU} / \mathrm{mL}$. Any colony count lower than this limit was rounded to $10^{2} \mathrm{CFU} / \mathrm{mL}$. All tests were performed in duplicate.
Table 1. Change in Colony Count $\left(\log _{10} \mathrm{CFU} / \mathrm{mL}\right)$ at 24 Hours Compared to Initial Inoculums for KPC-3-Producing K. Pneumoniae Isolates Using Time-Kill Methodology

\begin{tabular}{lcccc}
\hline Antibiotic & C16 & C17 & C38 & C50 \\
\hline Growth control & 5.05 & 4.13 & 4.78 & 4.55 \\
AMK & 1.36 & 3.75 & 2.98 & 4.23 \\
ETP & 4.00 & 3.14 & 5.16 & 4.83 \\
IPM & 4.02 & 3.30 & 4.50 & 4.71 \\
MEM & 3.69 & 3.59 & 4.90 & 4.09 \\
ETP + AMK & 1.57 & 2.81 & 3.86 & 4.45 \\
IPM + AMK & $-1.48^{*}$ & $-0.05^{*}$ & $-2.13^{*}$ & $-3.53^{*}$ \\
MEM + AMK & $-1.71^{*}$ & $-2.61^{*}$ & $-3.58^{*}$ & $-3.07^{*}$ \\
\hline
\end{tabular}

Synergy denoted as *.

AMK: amikacin; ETP: ertapenem; IPM: imipenem; MEM: meropenem.

\section{Results}

The mean starting inoculum for all of the time-kill experiments was $5.51 \times 10^{6} \mathrm{CFU} / \mathrm{mL}$ with an intra-day $\mathrm{CV}$ of $<$ $7.0 \%$. As monotherapy, none of the carbapenems nor AMK achieved and maintained bactericidal activity defined as $\geq$ $99.9 \%$ or $>3 \log _{10}$ killing (Table 1 ). Maximum killing of $\geq$ 90\% was observed for MEM and AMK against all isolates; and IPM against 3 isolates (except C17). ETP alone was least active as it did not achieve $\geq 90 \%$ killing in any isolates. Antibacterial activity was not maintained since significant regrowth was observed for all antibiotics after achieving $90 \%$ killing against each of the isolates (Fig. 1). In particular, amikacin demonstrated regrowth after 8 hrs.

Time-kill curve results for each carbapenem in combination with AMK are depicted in Figure 1. Synergy was defined as $\geq 2 \log _{10}$ decrease CFU/mL at $24 \mathrm{~h}$ for the combination when compared with the most active single agent of the combination, plus the number of surviving organisms for the antimicrobial combination was $\geq 2 \log _{10}$ less than the initial inoculum. Synergy was demonstrated for MEM and IPM in combination with AMK over the entire $24 \mathrm{~h}$ against all isolates. In addition, MEM and IPM with AMK achieved and maintained bactericidal activity ( $\geq 99.9 \%$ killing) at 24 h against 2 isolates (C38 and C50) and 1 (C50), respectively. For these isolates $\mathrm{C} 38$ and $\mathrm{C} 50$, the time to bactericidal activity were $12 \mathrm{~h}$ for MEM plus AMK and 24 and $8 \mathrm{~h}$, respectively, for IPM plus AMK.

Bactericidal activity and synergy were not observed for 

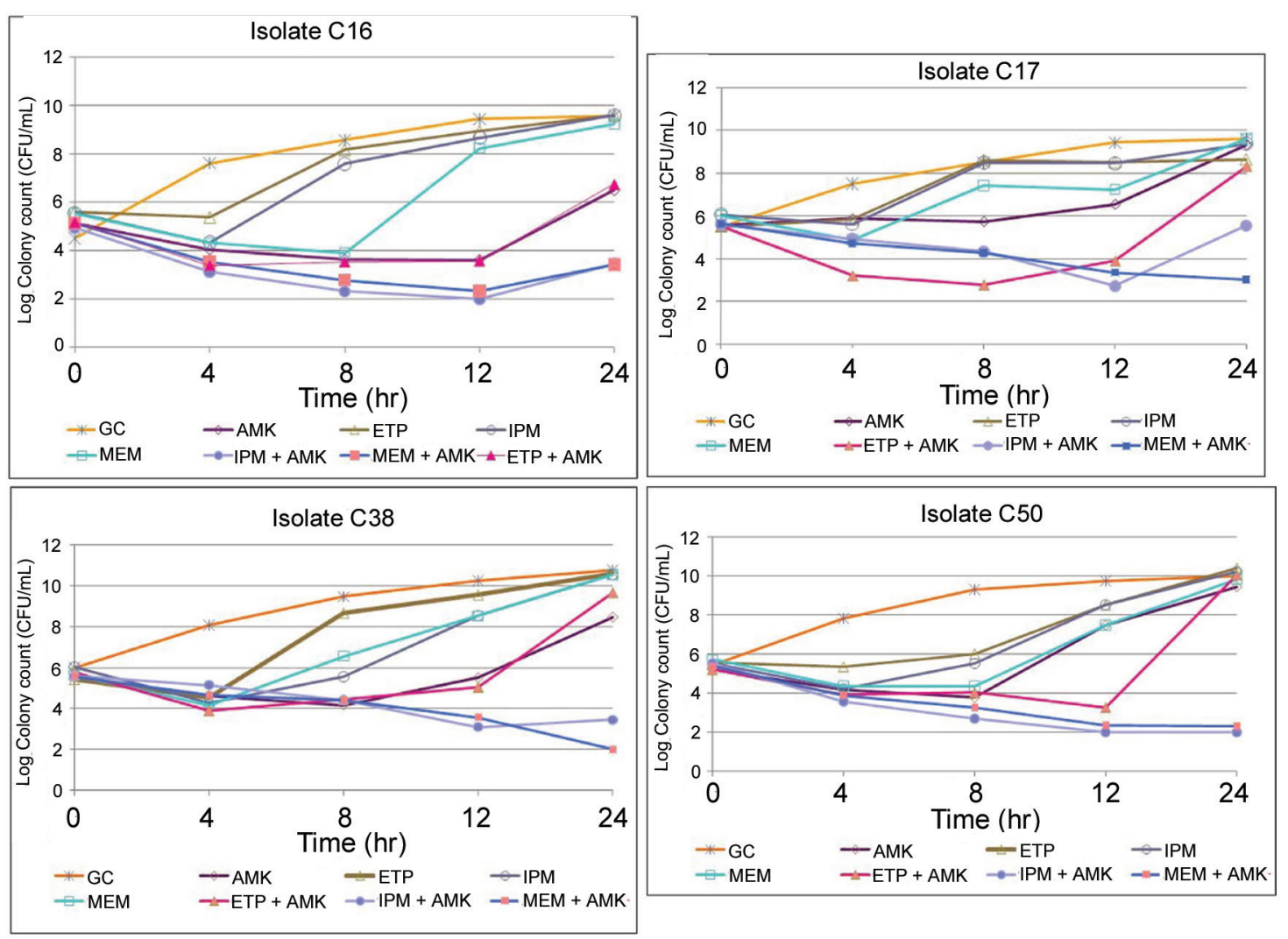

Figure 1. Time-kill studies of carbapenems and amikacin alone and in combination against four KPC-3-producing $K$. pneumoniae isolates. GC: Growth control; AMK: amikacin; ETP: ertapenem; IPM: imipenem; MEM: meropenem.

ETP combinations. In addition, significant regrowth for ETP with AMK was evident for all isolates between 8 and $12 \mathrm{~h}$. Slight regrowth occurred in 1 isolate (C16) for MEM and 3 isolates (except C50) for IPM combinations at $24 \mathrm{~h}$. None of the antibiotic combinations demonstrated antagonism, which was defined as $\geq 2 \log _{10}$ increase in colony count at $24 \mathrm{~h}$ for the combination when compared with the most active single agent of the combination.

\section{Discussion}

The clinical utility of carbapenem monotherapy is limited against isolates that produce KPC carbapenemases [13]. Studies of prolonged infusion of high-dose regimens of betalactams, particularly meropenem at $2 \mathrm{~g}$ every 8 hours over 3 -hours, against KPC isolates have produced conflicting results $[18,19]$. Although rapid colony reduction was initially observed, regrowth occurred after $6 \mathrm{hr}$ of meropenem exposure. Furthermore, meropenem exposure (or the percent time of free drug concentration above the MIC) for KPC isolates with elevated MICs of 8 and $16 \mu \mathrm{g} / \mathrm{mL}$ was significantly reduced due to rapid in vitro hydrolysis [19].

In light of the emerging resistance to carbapenems and the limited utility of overcoming this resistance through prolonged infusion of high-dose carbapenem monotherapy, combination therapy may play a role in the treatment of infections associated with KPC production since it may provide the potential for synergistic effects between two different classes of anti-infectives [20]. Studies evaluating antibiotic combinations against KPC-harboring isolates are limited. In our study, MEM or IPM in combination with AMK displayed synergy against KPC-producing K. pneumoniae. In another study, synergy was observed in combinations of polymyxin B with rifampin, doxycycline or tigecycline [7, 21].

\section{Conclusion}

The addition of AMK to carbapenems, particularly MEM and IPM, provided synergistic effects and enhanced bactericidal activity against $K$. pneumoniae with reduced susceptibility to carbapenems. Prolonged infusion of a high-dose carbapenem in combination with other potentially active antibiotics (such as AMK, tigecycline, and polymyxin B) may be a therapeutic option for serious infections associated with KPC production. Further investigation is warranted to determine if our in vitro observations translate into clinical efficacy. In addition, more research is imperative to optimize 
the dosing regimens of currently approved antibiotics using pharmacokinetic-pharmacodynamic principles and to explore the potential role of novel chemical entities.

\section{Acknowledgement and Transparency}

This study was supported by a grant from the American College of Clinical Pharmacy Research Institute. This work was presented at the 50th Interscience Conference on Antimicrobial Agents and Chemotherapy on September 2010 in Boston, MA. The authors disclose no potential conflict of interest relevant to this article. Dr. Le has served as advisor/ speaker and received research grants from Pfizer, Astellas and Cubist. Dr. Burgess has received research grants from AstraZenca, Ortho-McNeil, and Wyeth and served as advisor/speaker for Astellas, Forrest, and Wyeth.

\section{References}

1. Kitchel B, Rasheed JK, Patel JB, Srinivasan A, NavonVenezia S, Carmeli Y, Brolund A, et al. Molecular epidemiology of KPC-producing Klebsiella pneumoniae isolates in the United States: clonal expansion of multilocus sequence type 258. Antimicrob Agents Chemother 2009;53(8):3365-3370.

2. Rhomberg PR, Jones RN. Summary trends for the Meropenem Yearly Susceptibility Test Information Collection Program: a 10-year experience in the United States (1999-2008). Diagn Microbiol Infect Dis 2009;65(4):414-426.

3. Cordery RJ, Roberts CH, Cooper SJ, Bellinghan G, Shetty N. Evaluation of risk factors for the acquisition of bloodstream infections with extended-spectrum betalactamase-producing Escherichia coli and Klebsiella species in the intensive care unit; antibiotic management and clinical outcome. J Hosp Infect 2008;68(2):108115.

4. Gulmez D, Woodford N, Palepou MF, Mushtaq S, Metan G, Yakupogullari Y, Kocagoz S, et al. Carbapenemresistant Escherichia coli and Klebsiella pneumoniae isolates from Turkey with OXA-48-like carbapenemases and outer membrane protein loss. Int J Antimicrob Agents 2008;31(6):523-526.

5. Ktari S, Arlet G, Mnif B, Gautier V, Mahjoubi F, Ben Jmeaa M, Bouaziz M, et al. Emergence of multidrug-resistant Klebsiella pneumoniae isolates producing VIM-4 metallo-beta-lactamase, CTX-M-15 extended-spectrum beta-lactamase, and CMY-4 AmpC beta-lactamase in a Tunisian university hospital. Antimicrob Agents Chemother 2006;50(12):4198-4201.

6. Castanheira M, Sader HS, Deshpande LM, Fritsche
TR, Jones RN. Antimicrobial activities of tigecycline and other broad-spectrum antimicrobials tested against serine carbapenemase- and metallo-beta-lactamaseproducing Enterobacteriaceae: report from the SENTRY Antimicrobial Surveillance Program. Antimicrob Agents Chemother 2008;52(2):570-573.

7. Bratu S, Tolaney P, Karumudi U, Quale J, Mooty M, Nichani S, Landman D. Carbapenemase-producing Klebsiella pneumoniae in Brooklyn, NY: molecular epidemiology and in vitro activity of polymyxin B and other agents. J Antimicrob Chemother 2005;56(1):128132.

8. Zhanel GG, Baudry PJ, Tailor F, Cox L, Hoban DJ, Karlowsky JA. Determination of the pharmacodynamic activity of clinically achievable tigecycline serum concentrations against clinical isolates of Escherichia coli with extended-spectrum beta-lactamases, AmpC beta-lactamases and reduced susceptibility to carbapenems using an in vitro model. J Antimicrob Chemother 2009;64(4):824-828.

9. Nicasio AM, Crandon JL, Nicolau DP. In vivo pharmacodynamic profile of tigecycline against phenotypically diverse Escherichia coli and Klebsiella pneumoniae isolates. Antimicrob Agents Chemother 2009;53(7):27562761.

10. Bulik CC, Nicolau DP. In vivo efficacy of simulated human dosing regimens of prolonged-infusion doripenem against carbapenemase- producing Klebsiella pneumoniae. Antimicrob Agents Chemother 2010;54(10):41124115 .

11. Goossens H, Grabein B. Prevalence and antimicrobial susceptibility data for extended-spectrum betalactamase- and AmpC-producing Enterobacteriaceae from the MYSTIC Program in Europe and the United States (1997-2004). Diagn Microbiol Infect Dis 2005;53(4):257-264.

12. Jones RN, Mendes C, Turner PJ, Masterton R. An overview of the Meropenem Yearly Susceptibility Test Information Collection (MYSTIC) Program: 1997-2004. Diagn Microbiol Infect Dis 2005;53(4):247-256.

13. Deshpande LM, Rhomberg PR, Sader HS, Jones RN. Emergence of serine carbapenemases (KPC and SME) among clinical strains of Enterobacteriaceae isolated in the United States Medical Centers: report from the MYSTIC Program (1999-2005). Diagn Microbiol Infect Dis 2006;56(4):367-372.

14. Pattharachayakul S, Neuhauser MM, Quinn JP, Pendland SL. Extended-spectrum beta-lactamase (ESBL)producing Klebsiella pneumoniae: activity of single versus combination agents. J Antimicrob Chemother 2003;51(3):737-739.

15. Giamarellos-Bourboulis EJ, Grecka P, Giamarellou H. Comparative in vitro interactions of ceftazidime, meropenem, and imipenem with amikacin on multiresistant 
Pseudomonas aeruginosa. Diagn Microbiol Infect Dis 1997;29(2):81-86.

16. Le J, Castanheira M, Burgess DS, McKee B, Iqbal R, Jones RN. Clonal dissemination of Klebsiella pneumoniae carbapenemase KPC-3 in Long Beach, California. J Clin Microbiol 2010;48(2):623-625.

17. Yourassowsky E, Van der Linden MP, Crokaert F, Glupczynski Y. Effect of antibiotics carry-over on bacterial counting by 'spiral plating'. J Antimicrob Chemother 1988;21(1):138-140.

18. Crandon JL, Kuti JL, Jones RN, Nicolau DP. Comparison of 2002-2006 OPTAMA programs for US hospitals: focus on gram-negative resistance. Ann Pharmacother 2009;43(2):220-227.
19. Bulik CC, Christensen H, Li P, Sutherland CA, Nicolau DP, Kuti JL. Comparison of the activity of a human simulated, high-dose, prolonged infusion of meropenem against Klebsiella pneumoniae producing the KPC carbapenemase versus that against Pseudomonas aeruginosa in an in vitro pharmacodynamic model. Antimicrob Agents Chemother 2010;54(2):804-810.

20. Eliopoulos GaMRJ. Antimicrobial Combinations. 5th ed. Baltimore, MD: Lippincott Williams \& Wilkins, 2005.

21. Elemam A, Rahimian J, Doymaz M. In vitro evaluation of antibiotic synergy for polymyxin B-resistant carbapenemase-producing Klebsiella pneumoniae. J Clin Microbiol 2010;48(10):3558-3562. 